\title{
Early Postoperative Mortality Among Patients Aged 75 Years or Older With Stage II/III Rectal Cancer
}

\author{
Helmneh M. Sineshaw, MD, MPH ${ }^{1}$; K. Robin Yabroff, PhD ${ }^{1}$; V. Liana Tsikitis, MD, MCR ${ }^{2}$.
} Ahmedin Jemal, DVM, $\mathrm{PhD}^{1}$; and Timur Mitin, MD, $\mathrm{PhD}^{2}$

\begin{abstract}
Background: Elderly patients with rectal cancer have been excluded from randomized studies, thus little is known about their early postoperative mortality, which is critical for informed consent and treatment decisions. This study examined early mortality after surgery in elderly patients with locally advanced rectal cancer (LARC). Methods: Using the National Cancer Database, we identified patients aged $\geq 75$ years, diagnosed with clinical stage II/III rectal cancer who underwent surgery in 2004 through 2015. Descriptive analyses determined proportions and trends and multivariable logistic regression analyses were performed to determine factors associated with early mortality after rectal cancer surgery. Results: Among 11,794 patients with rectal cancer aged $\geq 75$ years, approximately $6 \%$ underwent local excision and $94 \%$ received radical resection. Overall 30-day, 90-day, and 6-month postoperative mortality rates were $4.2 \%, 7.8 \%$, and $11.5 \%$, respectively. Six-month mortality varied by age $(8.4 \%$ in age $75-79$ years to $18.3 \%$ in age $\geq 85$ years), and comorbidity score $(10.1 \%$ for comorbidity score 0 to $17.7 \%$ for comorbidity score $\geq 2$ ). Six-month mortality declined from $12.3 \%$ in 2004 through 2007 to $10.2 \%$ in 2012 through 2015 $\left(P_{\text {trend }}=.0035\right)$. Older age, higher comorbidity score, and lower facility case volume were associated with higher 6-month mortality. Patients treated at $\mathrm{NCl}$-designated centers had $30 \%$ lower odds of 6-month mortality compared with those treated at teaching/research centers. Conclusions: Six-month mortality rates after surgery among patients aged $\geq 75$ years with LARC have declined steadily over the past decade in the United States. Older age, higher comorbidity score, and care at a low-case-volume facility were associated with higher 6-month mortality after surgery. This information is necessary for informed consent and decisions regarding optimal management of elderly patients with LARC.
\end{abstract}

J Natl Compr Canc Netw 2020;18(4):443-451 doi: $10.6004 /$ jnccn.2019.7377

\footnotetext{
${ }^{1}$ American Cancer Society, Atlanta, Georgia; and ${ }^{2}$ Oregon Health and Science University, Portland, Oregon.
}

\section{Background}

Rectal cancer accounts for approximately one-third of patients newly diagnosed with colorectal cancer in the United States. ${ }^{1,2}$ The incidence of rectal cancer peaks at age $\geq 75$ years and its management is complex. ${ }^{3}$ Results of several important randomized clinical trials have shaped the current management of rectal cancer, which is built around trimodal therapy: preoperative chemoradiation therapy and total mesorectal excision (TME). ${ }^{4-6}$ Most randomized clinical trials, however, specifically exclude patients aged $>75$ years, ${ }^{6}$ complicating the application of randomized trial evidence in the management of elderly patients. The International Society of Geriatric Oncology issued a statement of recognition of special considerations and development of guidelines for elderly patients who are frequently excluded from many practiceestablishing large randomized clinical trials. ${ }^{7}$

Previously, we reported that trimodal therapy was associated with the best survival outcomes for patients diagnosed with locally advanced rectal cancer (LARC) in the United States using data from the National Cancer Database (NCDB) ${ }^{8}$ Patients who received concurrent chemoradiation therapy without surgery had lower survival rates. ${ }^{8}$ Others, however, used institutional data and reported that $>20 \%$ of patients may achieve clinical response (no observation of cancer) after undergoing chemoradiation therapy. These patients may consider a watchful waiting approach instead of immediate surgery, without worsening their survival outcomes. ${ }^{9,10}$ Observation after complete clinical response or transanal local excision of a small residual primary tumor avoiding extensive surgery is a viable clinical pathway gaining popularity in many countries, especially for elderly patients. ${ }^{11}$ However, treatment decision-making for these elderly patients is difficult. For example, a Dutch population-based study showed considerably higher 6-month mortality rates after TME among patients aged $\geq 75$ years $(14 \%)$

See JNCCN.org for supplemental online content. 
than in those aged $<75$ years $(4 \%) .{ }^{12}$ These observed higher rates may be attributed to potential clinical factors that, if addressed in a timely fashion, may decrease. Furthermore, clinical advances in elderly patient care and implementation of programs such as the American College of Surgeons National Surgical Quality Improvement Program may also decrease postoperative early-mortality rates. ${ }^{13}$ However, data are lacking on contemporary trends and factors associated with early mortality after surgery among this unique population of elderly patients with LARC in the United States. In this study, we fill this research gap by examining trends of and factors associated with early mortality after surgery in elderly patients with LARC in a large national sample. We hypothesize that there would be changes in early mortality over time, and age and comorbidity would be associated with early mortality differences after surgery among elderly patients with LARC.

\section{Methods}

\section{Data Source and Patient Cohorts}

We used data from the NCDB, a national, hospital-based cancer registry database jointly sponsored by the American College of Surgeons and the American Cancer Society, which captures approximately $70 \%$ of newly diagnosed cancer cases in the United States. ${ }^{14}$ We included patients diagnosed in 2004 through 2015 with single or first primary, invasive (behavior $=3$ ), primary site (C20.9), ICD-O-3 histology codes for rectal cancer (supplemental eTable 1, available with this article at JNCCN.org), AJCC clinical stage II or III (pathologic stage used if clinical stage was missing), and who were aged $\geq 75$ years and received surgical resection. Patients with missing/unknown values for insurance (including those with government insurance other than Medicare/Medicaid), area-level median income, chemotherapy, and radiotherapy were excluded. The final analytic cohort included 11,794 patients (Figure 1). Variables were coded according to the 2016 Facility Oncology Registry Data Standards Manual. ${ }^{15}$ Our study received exempt status from the Morehouse School of Medicine Institutional Review Board.

\section{Primary End Points}

Primary end points were 30-day, 90-day, and 6-month mortality from any cause among all patients with LARC after undergoing surgery.

\section{Independent Variables and Control Variables}

Our primary independent variable of interest was age at diagnosis, categorized as 75 to 79,80 to 84 , and $\geq 85$ years. We also evaluated comorbidity score and type of surgery (local excision, resection/total surgery). Local excision included local tumor excision, polypectomy, laser excision, or curette and fulguration; resection/total surgery included wedge or segmental resection, partial proctectomy, TME, total proctectomy, or total proctocolectomy. Comorbidity score was categorized as 0,1 , or $\geq 2$ based on the sum of weighted Charlson-Deyo score. ${ }^{16}$ Control variables were categorized as follows: race/ethnicity (non-Hispanic white, non-Hispanic black, Hispanic, other), sex (male, female), facility type (community cancer program, comprehensive community cancer program, teaching/research program, NCI-designed program/network, other programs), facility case volume (low, medium, high; ranked using tertiles by counting the number of cases reported by each facility during the study year by diagnosis year), arealevel median income quartiles $(<\$ 38,000, \$ 38,000-\$ 47,999$, $\$ 48,000-\$ 62,999, \geq \$ 63,000$ ), area-level median educational attainment $(\geq 21.0 \%, 13 \%-20.9 \%, 7.0 \%-12.9 \%$, or $<7.0 \%$ without high school diploma), clinical stage (II, III), and tumor size $(<2 \mathrm{~cm}, 2 \mathrm{~cm}$ to $<5 \mathrm{~cm}, \geq 5 \mathrm{~cm}$; size on pathologic report when patient did not receive radiation or systemic treatment before surgery, or size before surgery if patient received preoperative treatment). Facility case volume was categorized as low, medium, and high based on the number of rectal cancer cases reported by the facility over the study period by diagnosis year.

\section{Statistical Analysis}

We conducted descriptive analyses for patient characteristics by age group. We calculated the proportion of patients who died within 30 days, 90 days, and 6 months after surgical treatment by age, comorbidity score, facility type, and surgery type, and assessed trends using the Cochrane-Armitage trend test. Furthermore, we examined trends in 6-month mortality, adjusting for comorbidity score, because comorbidity score was significantly associated with trends in 6-month mortality.

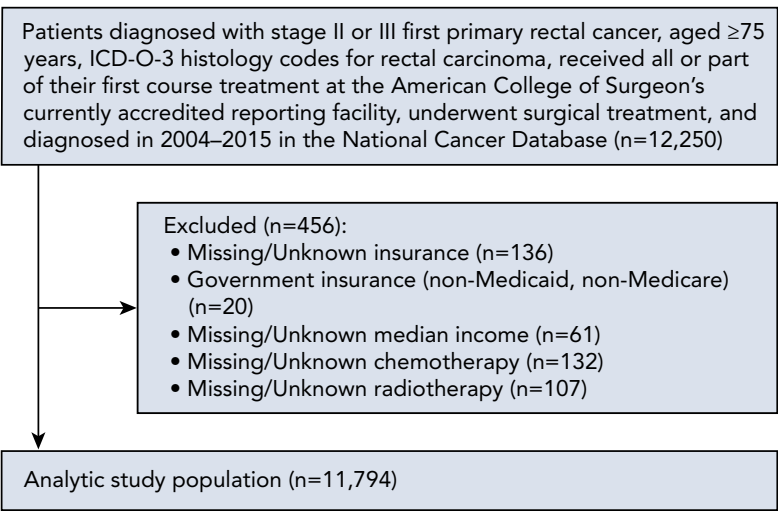

Figure 1. Flowchart of cohort inclusion/seclusion criteria for patients aged $\geq 75$ years diagnosed with stage II/III rectal cancer in the $\mathrm{Na}$ tional Cancer Database (2004-2015). 


\section{Table 1. Patient Characteristics}

\begin{tabular}{|c|c|c|c|c|c|}
\hline Variable & $\begin{array}{l}\text { Total } \\
\text { n (\%) }\end{array}$ & $\begin{array}{c}\text { Age } 75-79 \text { y } \\
n(\%)\end{array}$ & $\begin{array}{c}\text { Age 80-84 y } \\
n(\%)\end{array}$ & $\begin{array}{c}\text { Age } \geq 85 \text { y } \\
n(\%)\end{array}$ & $P$ Value \\
\hline Total, $n$ & 11,794 & 5,540 & 3,825 & 2,429 & \\
\hline Race/Ethnicity & & & & & $<.0001$ \\
\hline Non-Hispanic white & $9,437(80)$ & $4,365(46.3)$ & $3,060(32.4)$ & $2,012(21.3)$ & \\
\hline Non-Hispanic black & $637(5.4)$ & $338(53.1)$ & $210(33)$ & $89(14.0)$ & \\
\hline Hispanic & $523(4.4)$ & $279(53.3)$ & $161(30.8)$ & $83(15.9)$ & \\
\hline $2004-2007$ & $4,375(37.1)$ & $2,084(47.6)$ & $1,408(32.2)$ & $883(20.2)$ & \\
\hline $2008-2011$ & $3,776(32.0)$ & $1,746(46.2)$ & $1,228(32.5)$ & $802(21.2)$ & \\
\hline $2012-2015$ & $3,643(30.9)$ & $1,710(46.9)$ & $1,189(32.6)$ & $744(20.4)$ & \\
\hline Sex & & & & & $<.0001$ \\
\hline Male & $6,067(51.4)$ & $3,152(52)$ & $1,892(31.2)$ & $1,023(16.9)$ & \\
\hline III & $5,424(46.0)$ & $2,592(47.8)$ & $1,766(32.6)$ & $1,066(19.7)$ & \\
\hline Comorbidity score & & & & & .0470 \\
\hline 0 & $7,917(67.1)$ & $3,748(47.3)$ & $2,515(31.8)$ & $1,654(20.9)$ & \\
\hline 1 & $2,767(23.5)$ & $1,308(47.3)$ & $923(33.4)$ & $536(19.4)$ & \\
\hline$\geq 2$ & $1,110(9.4)$ & $484(43.6)$ & 387 (34.9) & $239(21.5)$ & \\
\hline Facility type & & & & & .0002 \\
\hline Community cancer program & $1,093(9.3)$ & $518(47.4)$ & $356(32.6)$ & $219(20)$ & \\
\hline Comprehensive community cancer program & $5,576(47.3)$ & $2,564(46.0)$ & $1,793(32.2)$ & $1,219(21.9)$ & \\
\hline Teaching/Research program & $2,351(19.9)$ & $1,120(47.6)$ & $764(32.5)$ & 467 (19.9) & \\
\hline $\mathrm{NCl}$-designated program & $920(7.8)$ & $491(53.4)$ & $290(31.5)$ & $139(15.1)$ & \\
\hline $38,000-47,999$ & $3,003(25.5)$ & $1,442(48)$ & $961(32)$ & $600(20)$ & \\
\hline $48,000-62,999$ & $3,259(27.6)$ & $1,501(46.1)$ & $1,060(32.5)$ & $698(21.4)$ & \\
\hline$\geq 63,000$ & $3,583(30.4)$ & $1,661(46.4)$ & $1,166(32.5)$ & $756(21.1)$ & \\
\hline No high school diploma, $\%^{c}$ & & & & & .008 \\
\hline$\geq 21.0$ & $1,948(16.5)$ & $980(50.3)$ & $606(31.1)$ & $362(18.6)$ & \\
\hline $13.0-20.9$ & $3,011(25.5)$ & $1,407(46.7)$ & $1,002(33.3)$ & $602(20)$ & \\
\hline $7.0-12.9$ & $4,059(34.4)$ & $1908(47)$ & $1,301(32.1)$ & $850(20.9)$ & \\
\hline$<7.0$ & $2,776(23.5)$ & $1,245(44.8)$ & $916(33)$ & $615(22.2)$ & \\
\hline Tumor size, $\mathrm{cm}$ & & & & & $<.0001$ \\
\hline$<2$ & $925(7.8)$ & $484(52.3)$ & $284(30.7)$ & $157(17)$ & \\
\hline 2 to $<5$ & $5,243(44.5)$ & $2,476(47.2)$ & $1,691(32.3)$ & $1,076(20.5)$ & \\
\hline$\geq 5$ & $4,088(34.7)$ & $1,767(43.2)$ & $1,350(33)$ & $971(23.8)$ & \\
\hline Missing/Unknown & $1,538(13)$ & $813(52.9)$ & $500(32.5)$ & $225(14.6)$ & \\
\hline Surgery & & & & & $<.0001$ \\
\hline Local excision & $720(6.1)$ & $234(32.5)$ & $231(32.1)$ & $255(35.4)$ & \\
\hline Resection/Total surgery & $1,1074(93.9)$ & $5,306(47.9)$ & $3,594(32.5)$ & $2,174(19.6)$ & \\
\hline
\end{tabular}

apathologic stage used if clinical stage was missing.

${ }^{b}$ Area-level median household income quartiles from the 2012 American Community Survey.

cArea-level quartiles for percentage of adults without a high school diploma from the 2012 American Community Survey. 
Table 2. Proportions of Early Postoperative Mortality

\begin{tabular}{|c|c|c|c|c|}
\hline Early Mortality & $\begin{array}{l}\text { Total } \\
\text { n (\%) }\end{array}$ & $\begin{array}{c}\text { Age 75-79 y } \\
n(\%)\end{array}$ & $\begin{array}{c}\text { Age 80-84 y } \\
n(\%)\end{array}$ & $\begin{array}{c}\text { Age } \geq 85 \text { y } \\
n(\%)\end{array}$ \\
\hline Overall, $n$ & 11,794 & 5,540 & 3,825 & 2,429 \\
\hline $30-d$ & $491(4.2)$ & $154(2.9)$ & $141(3.7)$ & $196(8.1)$ \\
\hline $90-d$ & $917(7.8)$ & $290(5.2)$ & $305(8.0)$ & $322(13.3)$ \\
\hline 6-mo & $1,352(11.5)$ & $466(8.4)$ & 441 (11.5) & 445 (18.3) \\
\hline \multicolumn{5}{|l|}{ Comorbidity score } \\
\hline $0, n$ & 7,917 & 3,748 & 2,515 & 1,654 \\
\hline $30-d$ & $286(3.6)$ & $88(2.4)$ & $82(3.3)$ & $116(7)$ \\
\hline $90-d$ & $536(6.8)$ & $171(4.6)$ & $174(6.9)$ & $191(11.6)$ \\
\hline 6-mo & $802(10.1)$ & $282(7.5)$ & $251(10)$ & $269(16.3)$ \\
\hline $1, n$ & 2,767 & 1,308 & 923 & 536 \\
\hline $30-d$ & $124(4.5)$ & $37(2.8)$ & $37(4)$ & $50(9.3)$ \\
\hline $90-d$ & $241(8.7)$ & $68(5.2)$ & $82(8.9)$ & $91(17)$ \\
\hline 6-mo & $254(9.2)$ & $111(8.5)$ & $123(13.3)$ & $120(22.4)$ \\
\hline$\geq 2, n$ & 1,110 & 484 & 387 & 239 \\
\hline $30-d$ & $81(7.3)$ & $29(6)$ & $22(5.7)$ & $30(12.6)$ \\
\hline $90-d$ & $140(12.6)$ & $51(10.5)$ & $49(12.7)$ & $40(16.7)$ \\
\hline 6-mo & $196(17.7)$ & $73(15.1)$ & $67(17.3)$ & $56(23.4)$ \\
\hline \multicolumn{5}{|l|}{ Facility type } \\
\hline Community cancer program, $\mathrm{n}$ & 1,093 & 518 & 356 & 219 \\
\hline $30-d$ & $60(5.5)$ & $22(4.3)$ & $17(4.8)$ & $21(9.6)$ \\
\hline $90-d$ & $107(9.8)$ & $39(7.5)$ & $34(9.6)$ & $34(15.5)$ \\
\hline 6-mo & $151(13.8)$ & $58(11.2)$ & $44(12.4)$ & $49(22.4)$ \\
\hline Comprehensive community cancer program, $\mathrm{n}$ & 5,576 & 2,564 & 1,793 & 1,219 \\
\hline $30-d$ & $265(4.8)$ & $84(3.3)$ & $76(4.2)$ & $105(8.6)$ \\
\hline $90-d$ & $475(8.5)$ & $147(5.7)$ & $160(8.9)$ & $168(13.8)$ \\
\hline 6-mo & $675(12.1)$ & $223(8.7)$ & $223(12.4)$ & $229(18.8)$ \\
\hline Teaching/Research program, n & 2,351 & 1,120 & 764 & 467 \\
\hline $30-d$ & $74(3.1)$ & $25(2.2)$ & $17(2.2)$ & $32(6.9)$ \\
\hline $90-d$ & $160(6.8)$ & $50(4.5)$ & $51(6.7)$ & $59(12.6)$ \\
\hline 6-mo & $260(11.1)$ & $91(8.1)$ & $87(11.4)$ & $82(17.6)$ \\
\hline $\mathrm{NCl}$-designated program, $\mathrm{n}$ & 920 & 491 & 290 & 139 \\
\hline $30-d$ & $19(2.1)$ & $5(1.0)$ & $8(2.8)$ & $6(4.3)$ \\
\hline $90-d$ & $42(4.6)$ & $12(2.4)$ & $17(5.9)$ & $13(9.4)$ \\
\hline 6-mo & $69(7.5)$ & $25(5.1)$ & $27(9.3)$ & $17(12.2)$ \\
\hline Other programs, $\mathrm{n}$ & 1,854 & 847 & 622 & 385 \\
\hline $30-d$ & $73(3.9)$ & $18(2.1)$ & $23(3.7)$ & $32(8.3)$ \\
\hline $90-d$ & $133(7.2)$ & $42(5.0)$ & $43(6.9)$ & $48(12.5)$ \\
\hline 6-mo & $197(10.6)$ & $69(8.2)$ & $60(9.7)$ & $68(17.7)$ \\
\hline \multicolumn{5}{|l|}{ Surgery type } \\
\hline Local excision, $\mathrm{n}$ & 720 & 234 & 231 & 255 \\
\hline $30-d$ & $22(3.1)$ & $8(3.4)$ & $2(0.9)$ & $12(4.7)$ \\
\hline $90-d$ & $50(6.9)$ & $12(5.1)$ & $16(6.9)$ & $22(8.6)$ \\
\hline 6-mo & $77(10.7)$ & $19(8.1)$ & $28(12.1)$ & $30(11.8)$ \\
\hline Resection/Total surgery, $n$ & 11,074 & 5,306 & 3,594 & 2,174 \\
\hline $30-d$ & $469(4.2)$ & $146(2.8)$ & $139(3.9)$ & $184(8.5)$ \\
\hline $90-d$ & $867(7.8)$ & $278(5.2)$ & $289(8)$ & $300(13.8)$ \\
\hline 6-mo & $1,275(11.5)$ & $447(8.4)$ & $413(11.5)$ & 415 (19.1) \\
\hline
\end{tabular}


We used multivariable logistic regression analysis to examine factors associated with 6-month mortality after surgery. Statistical significance was determined based on the 2-sided $P<.05$. All statistical analyses were conducted using SAS 9.4 (SAS Institute Inc.).

\section{Results}

Table 1 shows descriptive characteristics for the 11,794 patients in the study cohort. Of these, $47 \%$ were aged 75 to 79 years, $32.4 \%$ were aged 80 to 84 years, and $20.6 \%$ were aged $\geq 85$ years. Most were non-Hispanic white $(80 \%)$, with a higher proportion aged $\geq 85$ years compared with non-Hispanic black ( $21 \%$ vs $14 \%$ ). Nearly half $(47.3 \%)$ of all patients were treated in comprehensive community cancer programs versus only $7.8 \%$ treated at NCI-designated cancer programs. Most patients were treated in high-case-volume facilities (57.9\%).

The proportions of patients who experienced early postoperative mortality are displayed in Table 2 by age, comorbidity, facility type, and surgery type are displayed in Table 2. Overall proportions of 30-day, 90-day, and 6-month mortality were $4.2 \% 7.8 \%$, and $11.5 \%$, respectively. Sixmonth mortality varied by age group and comorbidity score, with the highest rate in the oldest age group ( $18.3 \%$ for age $\geq 85$ years vs $8.4 \%$ for age $75-79$ years) and among patients with higher comorbidity score $(17.7 \%$ for comorbidity score $\geq 2$ vs $10.1 \%$ for comorbidity score 0 ). There was marked difference in 6-month mortality by facility type among patients aged $\geq 85$ years, ranging from $12.2 \%$ in those treated at NCI-designated cancer programs to $22.4 \%$ in those treated at community cancer programs. Six-month mortality after local excision versus radical surgery was different only in the group of patients aged $\geq 85$ years $(11.8 \%$ vs $19.1 \%$, respectively).

Trends in 30-day or 90-day postoperative mortality did not change significantly. Six-month mortality steadily declined from $12.3 \%$ in $2004-2007$ to $10.2 \%$ in $2012-2015$ $\left(P_{\text {trend }}=.0035\right)$ (Figure 2, Table 3). Specifically, a significant decline in 6-month mortality occurred over time among patients aged 75 to 79 years, from $9.3 \%$ in $2004-2007$ to $6.8 \%$ in $2012-2015$ ( $P_{\text {trend }}=.0063$ ). A significant decline in 6-month mortality was also seen among patients with a comorbidity score of 1 (from $14.7 \%$ in $2004-2007$ to $9.8 \%$ in $2012-2015$; $P_{\text {trend }}=.0020$ ), those treated at comprehensive community cancer programs $\left(P_{\text {trend }}=.0420\right)$, and those who received surgical resection/total surgery $\left(P_{\text {trend }}=.0069\right)$. Six-month mortality rates declined significantly across age groups even after adjusting for comorbidity score (supplemental eTable 2).

Table 4 shows adjusted odds ratios (ORs) of 6-month postoperative mortality. Patients aged 80 to 84 years (OR, 1.42; 95\% CI, 1.24-1.63) and those $\geq 85$ years

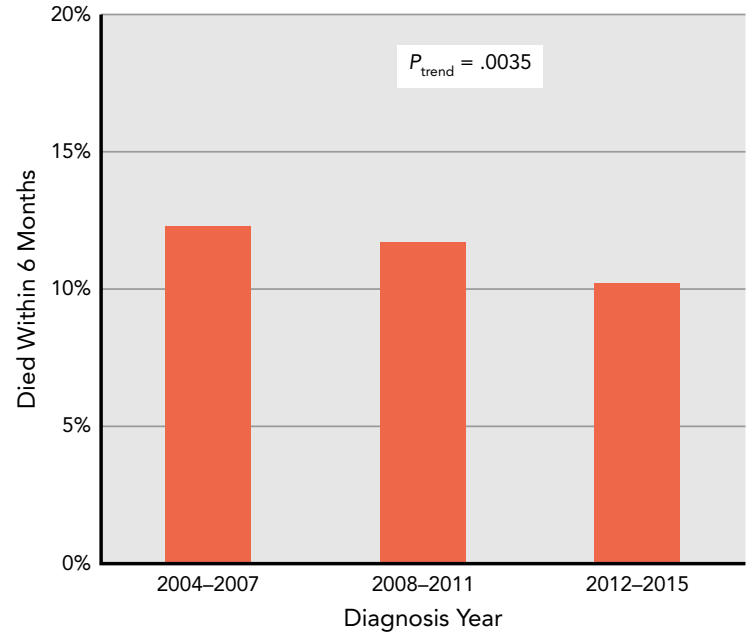

Figure 2. Trends in 6-month mortality among patients aged $\geq 75$ years diagnosed with locally advanced rectal cancer who underwent surgical treatment.

(OR, 2.51; 95\% CI, 2.17-2.89) had higher odds of 6-month mortality compared with those aged 75 to 79 years. Patients with a comorbidity score of $\geq 2$ had higher 6-month mortality (OR, 1.84; 95\% CI, 1.55-2.19) compared with those with no comorbid conditions. Patients treated at NCI-designated cancer programs had lower 6-month mortality (OR, 0.70; 95\% CI, 0.53-0.93) compared with those treated at teaching/research cancer programs. Treatments performed at low-volume facilities were also associated with higher 6-month mortality (OR, 1.23\%; 95\% CI, 1.08-1.40) compared with those performed at high-volume facilities.

\section{Discussion}

Trimodality therapy (preoperative chemoradiation therapy followed by TME) is the standard of care for management of patients with LARC, ${ }^{17}$ established by evidence from large prospective randomized clinical trials. ${ }^{4-6} \mathrm{Un}$ fortunately, patients aged $\geq 75$ years were specifically excluded from these trials, making the application of this standard of care in elderly patients less certain. Moreover, over the past decade the management of LARC has changed dramatically, with more personalized management based on tumor and patient characteristics. For patients with clinical response to neoadjuvant therapy, watchful waiting rather than immediate TME is gaining popularity based on large prospective studies. Omission of radiation therapy from preoperative treatment in select patients who experience response to chemotherapy is currently being studied in the large PROSPECT trial. ${ }^{18}$

To better inform patients about their treatment options, physicians need to know expected outcomes not only from randomized trials that include the most fit 
Table 3. Trends in Early Postoperative Mortality

\begin{tabular}{|lrrrrr} 
& \multicolumn{4}{c}{ Diagnosis Year, \% } & \\
\cline { 2 - 4 } Early Mortality & $\begin{array}{c}\mathbf{2 0 0 4}- \\
\mathbf{2 0 0 7}\end{array}$ & $\mathbf{2 0 0 8 -}$ & $\mathbf{2 0 1 2 -}$ & $\mathbf{2 0 1 5}$ & $\boldsymbol{P}_{\text {trend }}$ \\
\hline Overall & 4.1 & 4.3 & 4.0 & .8433 \\
\hline $30-d$ & 8.3 & 7.7 & 7.3 & .0784 \\
\hline 90-d & 12.3 & 11.7 & 10.2 & .0035 \\
\hline 6-mo & & & &
\end{tabular}

Age group

75-79 y

\begin{tabular}{lrrrr}
\hline $30-\mathrm{d}$ & 2.8 & 3.1 & 2.5 & .5787 \\
\hline $90-\mathrm{d}$ & 5.9 & 5.6 & 4.2 & .0216 \\
\hline $6-\mathrm{mo}$ & 9.3 & 8.9 & 6.8 & .0063 \\
\hline $80-84 \mathrm{y}$ & & & & \\
\hline $30-\mathrm{d}$ & 3.6 & 3.3 & 4.1 & .5261 \\
\hline $90-\mathrm{d}$ & 8.3 & 7.2 & 8.4 & .9732 \\
\hline $6-\mathrm{mo}$ & 12.4 & 10.8 & 11.3 & .3320 \\
\hline$\geq 85 \mathrm{y}$ & & & & \\
\hline $30-\mathrm{d}$ & 8.2 & 8.5 & 7.5 & .6625 \\
\hline $90-\mathrm{d}$ & 14.0 & 13.1 & 12.5 & .3568 \\
\hline $6-\mathrm{mo}$ & 19.3 & 19.1 & 16.4 & .1477
\end{tabular}

Comorbidity score

0

\begin{tabular}{lrrrr}
\hline $30-d$ & 3.4 & 3.7 & 3.8 & .4193 \\
\hline $90-d$ & 7.1 & 6.6 & 6.5 & .3658 \\
\hline $6-m o$ & 10.9 & 10.1 & 9.3 & .0601
\end{tabular}

1

\begin{tabular}{lrrrr}
\hline $30-d$ & 5.1 & 4.6 & 3.6 & .1347 \\
\hline $90-d$ & 10.1 & 8.5 & 7.1 & .0248 \\
\hline 6-mo & 14.7 & 13.3 & 9.8 & .0020 \\
\hline 2 & & & & \\
\hline $30-d$ & 6.9 & 7.9 & 7.0 & .9162 \\
\hline $90-d$ & 11.9 & 12.7 & 13.4 & .5472 \\
\hline 6-mo & 16.5 & 18.3 & 18.5 & .4513
\end{tabular}

Facility type

Community cancer program

\begin{tabular}{lrrrr}
\hline $30-d$ & 5.7 & 6.8 & 3.6 & .3058 \\
\hline $90-d$ & 10.7 & 11.7 & 6.0 & .0663 \\
\hline $6-m o$ & 14.4 & 15.7 & 10.6 & .2156
\end{tabular}

Comprehensive community cancer program

\begin{tabular}{lrrrr|}
\hline $30-d$ & 4.6 & 4.6 & 5.0 & .5915 \\
\hline $90-d$ & 9.3 & 7.9 & 8.3 & .2320 \\
\hline $6-m o$ & 13.4 & 11.3 & 11.3 & .0420 \\
\hline Teaching/Research program & & & & \\
\hline $30-d$ & 4.0 & 2.0 & 3.4 & .4535 \\
\hline $90-d$ & 7.8 & 6.1 & 6.5 & .3049 \\
\hline 6-mo & 12.4 & 11.2 & 9.5 & .0626 \\
\hline
\end{tabular}

(continued)

\begin{tabular}{|c|c|c|c|c|}
\hline \multirow[b]{2}{*}{ Early Mortality } & \multicolumn{3}{|c|}{ Diagnosis Year, \% } & \multirow[b]{2}{*}{$\boldsymbol{P}_{\text {trend }}$} \\
\hline & $\begin{array}{c}2004- \\
2007\end{array}$ & $\begin{array}{c}2008- \\
2011\end{array}$ & $\begin{array}{c}2012- \\
2015\end{array}$ & \\
\hline \multicolumn{5}{|c|}{$\mathrm{NCl}$-designated program } \\
\hline $30-d$ & 1.8 & 2.2 & 2.3 & .6789 \\
\hline $90-d$ & 4.5 & 4.0 & 5.1 & .6927 \\
\hline 6-mo & 8.7 & 6.9 & 6.8 & .3541 \\
\hline \multicolumn{5}{|c|}{ Other programs } \\
\hline $30-d$ & 2.8 & 5.8 & 3.1 & .6695 \\
\hline 90-d & 6.1 & 8.4 & 7.0 & .5106 \\
\hline 6-mo & 9.3 & 13.0 & 9.5 & .7666 \\
\hline \multicolumn{5}{|l|}{ Surgery type } \\
\hline \multicolumn{5}{|l|}{ Local excision } \\
\hline $30-d$ & 4.2 & 4.1 & 0.5 & .0245 \\
\hline $90-d$ & 7.6 & 7.8 & 5.2 & .3293 \\
\hline 6-mo & 12.5 & 10.3 & 9.0 & .2089 \\
\hline \multicolumn{5}{|c|}{ Resection/Total surgery } \\
\hline $30-d$ & 4.1 & 4.3 & 4.3 & .7832 \\
\hline $90-d$ & 8.3 & 7.7 & 7.4 & .1141 \\
\hline 6-mo & 12.3 & 11.8 & 10.3 & .0069 \\
\hline
\end{tabular}

and generally younger patients but also from real-practice data in elderly patients with comorbid conditions. Our study provides information about 30-day, 90-day, and 6 -month mortality following surgery for LARC among the unique population of elderly patients aged $\geq 75$ years. Mortality is likely the result of causes other than rectal cancer progression, because patients with stage II or III rectal cancer selected for surgery generally undergo a thorough evaluation to rule out the presence of distant metastasis. Development of metastatic disease, even if it occurs within 6 months of surgery, is unlikely to lead to rectal cancer-related mortality. Moreover, the mortality rates that we report do not solely reflect surgical mortality, but instead reflect risk of death from a complex interplay of advanced age, comorbid conditions, and the impact of a major surgery on these patients; this is supported by comparison of relatively low 30- and 90-day mortality versus the more substantial 6-month mortality.

We observed a significant declining trend in 6-month postoperative mortality among patients aged $\geq 75$ years with LARC in the United States during the most recent period, which may in part reflect improvements in quality of care over the past decade. ${ }^{19}$ Advances in surgical skills and facilities, better anesthesia, fewer emergency procedures, improved access to healthcare services, and greater availability of effective treatments 


\section{Table 4. Adjusted ORs Determining Odds of 6-Month Postoperative Mortality}

Variable OR $(95 \% \mathrm{Cl})^{\mathrm{a}}$

Age group, y

\begin{tabular}{lc}
$75-79$ & Ref \\
\hline $80-84$ & $1.42(1.24-1.63)$ \\
$\geq 85$ & $2.51(2.17-2.89)$
\end{tabular}

Race/Ethnicity

Non-Hispanic white

Ref

Non-Hispanic black

$1.16(0.90-1.51)$

Hispanic

$1.06(0.79-1.42)$

Other/Unknown

$1.16(0.96-1.39)$

Diagnosis year

2004-2007

Ref

2008-2011

$0.95(0.82-1.10)$

2012-2015

$0.82(0.70-0.96)$

Sex
Female

Ref

Male

$1.26(1.12-1.42)$

Clinical stage ${ }^{b}$

\begin{tabular}{cc}
\hline II & Ref \\
\hline III & $1.09(0.97-1.22)$
\end{tabular}

Tumor size, $\mathrm{cm}$

$<2$

Ref

2 to $<5$

$1.09(0.86-1.39)$

$\geq 5$

1.35 (1.06-1.72)

Missing/Unknown

$1.14(0.86-1.50)$

Comorbidity score

\begin{tabular}{lc}
\hline 0 & Ref \\
\hline 1 & $1.30(1.13-1.48)$ \\
$\geq 2$ & $1.84(1.55-2.19)$
\end{tabular}

Facility type

Teaching/Research program

Ref

Community cancer program

$1.17(0.93-1.47)$

Comprehensive community cancer program $1.06(0.90-1.23)$

$\mathrm{NCl}$-designated program

Other programs

$0.92(0.75-1.12)$

Facility case volume

High

Ref

Medium

$1.10(0.89-1.35)$

Low

Median income, \$USD

$\geq 63,000$

Ref

$<38,000$

$1.09(0.87-1.37)$

38,000-47,999

$1.18(0.98-1.42)$

$48,000-62,999$

$1.10(0.93-1.31)$
$0.70(0.53-0.93)$

$1.23(1.08-1.40)$

\begin{tabular}{|c|c|}
\hline Variable & OR $(95 \% \mathrm{Cl})^{\mathrm{a}}$ \\
\hline \multicolumn{2}{|c|}{ No high school diploma, \% } \\
\hline$<7$ & Ref \\
\hline $7.0-12.9$ & $0.97(0.82-1.15)$ \\
\hline 13.0-20.9 & $0.95(0.77-1.16)$ \\
\hline$\geq 21.0$ & $0.98(0.77-1.24)$ \\
\hline
\end{tabular}

Abbreviation: $O R$, odds ratio.

aAdjusted for age, race/ethnicity, diagnosis year, sex, clinical stage, tumor size, comorbidity score, facility type, facility case volume, area-level median income, area-level percent of persons with no high school diploma.

bPathologic stage used if clinical stage was missing.

'Area-level median household income quartiles from the 2012 American Community Survey.

${ }^{\mathrm{d}}$ Area-level quartiles for percentage of adults without a high school diploma from the 2012 American Community Survey.

and postoperative care could contribute to this declining trend. ${ }^{20-22}$ Furthermore, we found that the largest significant decline in 6-month mortality was in patients with lower comorbidity score, those treated at comprehensive community cancer programs, those who received surgical resection/total surgery, and those aged 75 to 79 years. Advances in perioperative management may allow patients in this group to receive the standard of care and expect similar outcomes to patients enrolled in large randomized clinical trials. Lower comorbidity score and relatively younger age among these elderly patients could also lead to a favorable assessment for preoperative treatment, which may increase the chance of surgical resection/total surgery and improve early mortality. In a previous study from Europe, Rutten et $\mathrm{al}^{12}$ did not find decreases in postoperative mortality over time among patients aged $\geq 75$ years with LARC. In contrast, our findings suggest progress toward reducing postoperative mortality and future opportunities to mitigate early postoperative mortality. Although more evidence is needed, some of these elderly patients could receive standard of care, and those with more advanced age or higher comorbidity score could be counseled regarding alternative treatment options, such as watchful waiting in the case of clinical response to preoperative treatment or local excision of residual lesions-a practice that has been adopted in some European centers.

Our findings of significant associations between early mortality and cancer treatment facility type and case volume correspond with those of other studies. ${ }^{23,24}$ After accounting for differences in age composition and other factors, we found that patients treated at highvolume and NCI-designated facilities had lower odds of early postoperative mortality, suggesting that expert care provided by surgical teams, nursing staff, and rehabilitation programs at these facilities may account 
for better outcomes in elderly patients with medical comorbidities. Efforts such as implementation of surgical quality improvement programs, care coordination, or streamlining referral systems to centers of excellence that improve access to high-quality care could lower early postoperative mortality in this group of patients. ${ }^{13,25}$ In addition, patients with rectal cancer require specialized management, and centralizing care could improve postoperative outcome.

A strength of our study is the use of a large contemporary nationwide oncologic outcomes database, which includes $70 \%$ of patients newly diagnosed with cancer in the United States. The NCDB also implements stringent data quality and ascertainment methods. ${ }^{15}$ We acknowledge several limitations as well. The NCDB is hospital-based, and participating hospitals may differ from those that do not participate; therefore, findings may lack generalizability. In addition, the NCDB does not collect information on cause of death, and all estimates are based on all-cause mortality. We were also unable to account for performance status, quality of life, symptoms, postoperative complications, and patient/physician preferences that may influence treatment decisions, because the NCDB does not capture these variables.

\section{Conclusions}

Although 6-month postoperative mortality among patients aged $\geq 75$ years with LARC declined steadily in the United States over the past decade, the odds of postsurgical mortality remains a significant challenge in management of these patients. In addition to older age and higher comorbidity score, receipt of treatment at lowvolume or non-NCI-designated facilities were associated with higher 6-month postoperative mortality. Improving prevention and management of comorbid conditions and specialized postoperative care could improve surgical outcome in the oldest of these patients. To achieve the best outcomes, treatment recommendations provided by expert multidisciplinary care providers could be personalized to individual patients, and applying aggressive surgical treatment should be approached with great care in patients aged $\geq 75$ years with LARC. More innovative research is needed pertaining to early postoperative mortality in patients aged $\geq 75$ years, using observational data because these patients are excluded from randomized control trials.
Submitted August 19, 2019; accepted for publication October 31, 2019.

Previous presentation: Preliminary findings were presented at the 2019 ASCO Annual Meeting; June 3, 2019; Chicago, Illinois. Abstract 248295.

Author contributions: Study concept and design: All authors. Data acquisition: Sineshaw. Data interpretation: All authors. Drafting of manuscript Sineshaw, Mitin. Critical revision of manuscript for important intellectual content: All authors. Administrative, technical, or material support: Sineshaw, Yabroff, Jemal. Study supervision: Sineshaw, Yabroff, Jemal, Mitin.

Disclosures: Dr. Mitin has disclosed that he has received grant/research support from Novocure, Inc; is a scientific advisor for Novocure and Janssen and receives royalties from UpToDate, Inc. The remaining authors have disclosed that they have not received any financial consideration from any person or organization to support the preparation, analysis, results, or discussion of this article.

Funding: This work was supported by the American Cancer Society Intramural Research (no grant number applicable to H.M.S., Y.K.R., and A.J.).

Disclaimer: The data used in the study are derived from a limited data set of the National Cancer Database. The authors acknowledge the efforts of the American College of Surgeons, the Commission on Cancer, and the American Cancer Society in the creation of the National Cancer Database. The American College of Surgeons and the Commission on Cancer have not verified and are not responsible for the analytic or statistical methodology used, or the conclusions drawn from these data by the authors.

Correspondence: Helmneh M. Sineshaw, MD, MPH, American Cancer Society, 250Williams Street NW, Atlanta, GA 30303. Email: helmneh.sineshaw@cancer.org

\section{References}

1. Siegel RL, Miller KD, Jemal A. Cancer statistics, 2019. CA Cancer J Clin 2019;69:7-34

2. Siegel RL, Miller KD, Fedewa SA, et al. Colorectal cancer statistics, 2017 CA Cancer J Clin 2017;67:177-193.

3. Siegel RL, Fedewa SA, Anderson WF, et al. Colorectal cancer incidence patterns in the United States, 1974-2013. J Natl Cancer Inst 2017;109.

4. Bosset JF, Calais G, Mineur L, et al. Fluorouracil-based adjuvant chemotherapy after preoperative chemoradiotherapy in rectal cancer: longterm results of the EORTC 22921 randomised study. Lancet Oncol 2014; 15:184-190.

5. Gérard JP, Conroy T, Bonnetain F, et al. Preoperative radiotherapy with or without concurrent fluorouracil and leucovorin in T3-4 rectal cancers: results of FFCD 9203. J Clin Oncol 2006;24:4620-4625.

6. Sauer R, Becker H, Hohenberger W, et al. Preoperative versus postoperative chemoradiotherapy for rectal cancer. N Engl J Med 2004;351: 1731-1740.

7. Papamichael D, Audisio RA, Glimelius B, et al. Treatment of colorectal cancer in older patients: International Society of Geriatric Oncology (SIOG) consensus recommendations 2013. Ann Oncol 2015;26:463-476.

8. Sineshaw HM, Jemal A, Thomas CR Jr, et al. Changes in treatment patterns for patients with locally advanced rectal cancer in the United States over the past decade: an analysis from the National Cancer Data Base. Cancer 2016;122:1996-2003.
9. Smith JD, Ruby JA, Goodman KA, et al. Nonoperative management of rectal cancer with complete clinical response after neoadjuvant therapy. Ann Surg 2012;256:965-972.

10. Habr-Gama A, Perez RO, Nadalin W, et al. Operative versus nonoperative treatment for stage 0 distal rectal cancer following chemoradiation therapy: long-term results. Ann Surg 2004;240:711-717 [discussion: 717-718].

11. Bujko K, Glynne-Jones R, Papamichael D, et al. Optimal management of localized rectal cancer in older patients. J Geriatr Oncol 2018;9:696-704.

12. Rutten $H$, den Dulk $M$, Lemmens $V$, et al. Survival of elderly rectal cancer patients not improved: analysis of population based data on the impact of TME surgery. Eur J Cancer 2007;43:2295-2300.

13. Hall BL, Hamilton BH, Richards $K$, et al. Does surgical quality improve in the American College of Surgeons National Surgical Quality Improvement Program: an evaluation of all participating hospitals. Ann Surg 2009;250: 363-376.

14. Mallin K, Browner A, Palis B, et al. Incident cases captured in the Nationa Cancer Database Compared with those in U.S. population based central cancer registries in 2012-2014. Ann Surg Oncol 2019;26:1604-1612.

15. Commission on Cancer. FORDS: Facility Oncology Registry Data Standards: Revised for 2016. Accessed April 18, 2019. Available at: https:// www.facs.org/-/media/files/quality-programs/cancer/ncdb/fords-2016. ashx 
16. Al-Refaie WB, Parsons HM, Habermann EB, et al. Operative outcomes beyond 30-day mortality: colorectal cancer surgery in oldest old. Ann Surg 2011;253:947-952.

17. Benson AB III, Venook AP, Al-Hawary MM, et al. NCCN Clinical Practice Guidelines in Oncology: Rectal Cancer. Version 1.2020. Accessed February 20, 2020. To view the most recent version, visit NCCN.org

18. Schrag D, Weiser M, Saltz $L$, et al. Challenges and solutions in the design and execution of the PROSPECT Phase II/III neoadjuvant rectal cancer trial (NCCTG N1048/Alliance). Clin Trials 2019;16:165-175.

19. Cohen ME, Liu Y, Ko CY, et al. Improved surgical outcomes for ACS NSQIP hospitals over time: evaluation of hospital cohorts with up to 8 years of participation. Ann Surg 2016;263:267-273.

20. Evans MD, Thomas R, Williams GL, et al. A comparative study of colorectal surgical outcome in a national audit separated by 15 years. Colorectal Dis 2013;15:608-612.
21. Arezzo A, Passera R, Scozzari G, et al. Laparoscopy for rectal cancer reduces short-term mortality and morbidity: results of a systematic review and meta-analysis. Surg Endosc 2013;27:1485-1502.

22. Tejedor P, Pastor C, Gonzalez-Ayora S, et al. Short-term outcomes and benefits of ERAS program in elderly patients undergoing colorectal surgery: a case-matched study compared to conventional care. Int J Colorectal Dis 2018;33:1251-1258.

23. Birkmeyer JD, Lucas FL, Wennberg DE. Potential benefits of regionalizing major surgery in Medicare patients. Eff Clin Pract 1999;2:277-283.

24. Begg CB, Cramer LD, Hoskins WJ, et al. Impact of hospital volume on operative mortality for major cancer surgery. JAMA 1998;280: 1747-1751.

25. Kwon DH, Tisnado DM, Keating NL, et al. Physician-reported barriers to referring cancer patients to specialists: prevalence, factors, and association with career satisfaction. Cancer 2015;121:113-122. 
Supplemental online content for:

\section{Early Postoperative Mortality Among Patients Aged 75 Years or Older With Stage II/III Rectal Cancer}

Helmneh M. Sineshaw, MD, MPH; K. Robin Yabroff, PhD; V. Liana Tsikitis, MD, MCR;

Ahmedin Jemal, DVM, PhD; and Timur Mitin, MD, PhD

J Natl Compr Canc Netw 2020;18(4):443-451

eTable 1: Rectal Cancer Topography and Histology Codes

eTable 2: Comorbidity Score-Adjusted ORs Determining Trends in Odds of 6-Month Postoperative Mortality 


\section{eTable 1. Rectal Cancer Topography and Histology Codes}

ICD-0-3 Topography Codes

C20.9

\section{ICD-0-3 Histology Codes}

$8000,8001,8002,8003,8004,8005,8010,8011,8012,8013,8014,8015,8020,8021,8022,8030,8031,8032,8033,8034$, $8035,8041,8043,8050,8051,8052,8070,8071,8072,8073,8074,8075,8076,8078,8120,8121,8122,8123,8124,8140$, $8141,8143,8145,8147,8210,8211,8220,8221,8230,8231,8255,8260,8261,8262,8263,8430,8440,8480,8481,8490$, $8510,8550,8551,8560,8562,8570,8571,8572,8573,8574,8575,8576$

\begin{tabular}{|lccc|}
\hline eTable 2. Comorbidity Score-Adjusted ORs \\
Determining Trends in Odds of 6-Month \\
Postoperative Mortality
\end{tabular}

Abbreviation: OR, odds ratio.

${ }^{a}$ Adjusted for comorbidity score. 